\title{
ON THE EXISTENCE OF MAXIMAL AND MINIMAL SOLUTIONS FOR PARABOLIC PARTIAL DIFFERENTIAL EQUATIONS
}

\author{
J. W. BEBERNES AND K. SCHMITT
}

\begin{abstract}
The existence of maximal and minimal solutions for initialboundary value problems and the Cauchy initial value problem associated with $L u=f(x, t, u, \nabla u)$ where $L$ is a second order uniformly parabolic differential operator is obtained by constructing maximal and minimal solutions from all possible lower and all possible upper solutions, respectively. This approach allows $f$ to be highly nonlinear, i.e., $f$ locally Hölder continuous with almost quadratic growth in $|\nabla u|$.
\end{abstract}

1. Introduction. In this paper we study the existence of maximal and minimal solutions for initial boundary value problems and the Cauchy initial value problem associated with

$$
L u=f(x, t, u, \nabla u),
$$

where $L$ is a second order uniformly parabolic differential operator.

In recent years a considerable amount of study has been devoted to establish the existence of solutions for elliptic and parabolic problems provided upper and lower solutions of such problems exist. Much of this work has its basis in the fundamental paper of Nagumo [9] as carried further by Akǒ [1] and Tomi [17]. Keller [5] and Amann [2] constructed solutions between upper and lower solutions of elliptic problems using a monotone iteration scheme which was possible because of certain one sided Lipschitz continuity assumptions upon the nonlinear terms and because the nonlinearities were assumed gradient independent. (For a recent survey of such results see the paper by Schmitt [16].) Sattinger [15] extended Amann's results (assuming that $f(x, t, u)$ is $C^{1}$ with respect to $u$ ) to parabolic initial boundary value problems using a similar monotone iteration scheme. This work was subsequently extended to various kinds of problems for both elliptic and parabolic equations by Pao [10], [11] and Puel [13] using either monotone iteration techniques or the theory of monotone operators.

In these papers a minimal solution is produced by starting the iteration scheme with the given lower solution and the maximal solution by commencing the scheme with the upper solution. While this procedure has certain

Received by the editors July 18, 1977 and, in revised form, March 16, 1978.

AMS (MOS) subject classifications (1970). Primary 35K55; Secondary 35B45, 34G05.

Key words and phrases. Maximal solutions, parabolic partial differential equations, lower solutions, nonuniqueness. 
computational advantages the permissible class of nonlinearities is restricted to those $f$ described above.

Using a different approach (an approach patterned after methods employed by Akǒ [1] in the study of Dirichlet boundary value problems for elliptic equations) we demonstrate the existence of maximal and minimal solutions for both initial boundary value and Cauchy initial value problems associated with (P) where a much larger class of nonlinearities $f$ is permissible, e.g., $f$ may depend upon $\nabla u$ (though it is necessary to require a Nagumo type growth condition with respect to this variable) and $f$ need not be Lipschitz continuous with respect to $u$. The maximal and minimal solutions will, respectively, be obtained as the supremum of all possible lower solutions and the infinimum of all possible upper solutions where the concept of upper and lower solutions as used here is somewhat more general than that used in the papers quoted above (see definitions below).

In the next section we make precise our assumptions and formulate the problems to be considered. In $\$ 3$ we state an invariance result from which we deduce the existence of solutions lying between upper and lower solutions while in $\$ 4$ we state and prove our main result.

2. Definitions and notation. Let $\mathbf{R}^{n}$ denote $n$-dimensional real Euclidean space with norm denoted by $|\cdot|$, let $\Omega$ be a bounded domain in $\mathbf{R}^{n}$ whose boundary $\partial \Omega$ is an $n-1$ dimensional manifold of class $C^{2+\alpha}, 0<\alpha<1$. Let $B^{N}=\left\{x \in \mathbf{R}^{n}:|x|<N\right\}, \pi_{T}=\Omega \times(0, T), \pi_{T}^{N}=B^{N} \times(0, T), \Gamma_{T}=$ $(\partial \Omega \times[0, T]) \cup(\bar{\Omega} \times\{0\}), \Gamma_{T}^{N}=\left(\partial B^{N} \times[0, T]\right) \times\left(\bar{B}^{N} \times\{0\}\right)$.

For $u: \bar{D} \times[0, T] \rightarrow \mathbf{R}\left(D\right.$ will equal either $\Omega$ or $\mathbf{R}^{n}$, depending on which problem is being considered), define $L u$ by

$$
(L u)(x, t)=\sum_{i, j=1}^{n} a_{i j}(x, t) \frac{\partial^{2} u}{\partial x_{i} \partial x_{j}}+\sum_{i=1}^{n} b_{i}(x, t) \frac{\partial u}{\partial x_{i}}+c(x, t) u-\frac{\partial u}{\partial t},
$$

where $a_{i j}, b_{i}, c \in C^{\alpha, \alpha / 2}(\bar{D} \times[0, T]), 0<\alpha<1,\left(C^{\alpha, \alpha / 2}(\cdot)\right.$ shall denote the usual Hölder space of functions $u(x, t)$ which are Hölder continuous with respect to $x$ with exponent $\alpha$ and with respect to $t$ with exponent $\alpha / 2$ and which is endowed with the standard Hölder norm, see [6]), $1 \leqslant i, j \leqslant n$, and $c \leqslant 0$. We assume that $L$ is uniformly parabolic, i.e., there exist constants $\mu$, $\lambda, 0<\lambda<\mu$, such that for all $\xi \in \mathbf{R}^{n}$ and all $(x, t) \in \bar{D} \times[0, T]$

$$
\lambda|\xi|^{2} \leqslant \sum_{i, j=1}^{n} a_{i j}(x, t) \xi_{i} \xi_{j} \leqslant \mu|\xi|^{2} .
$$

Let $f: \bar{D} \times[0, T] \times \mathbf{R} \times \mathbf{R}^{n} \rightarrow \mathbf{R}$, defined by $(x, t, u, p) \mapsto f(x, t, u, p)$ be a Hölder continuous function with Hölder exponents $\alpha, \alpha / 2, \alpha, \alpha$ in the respective variables $x, t, u, p$.

Given $\psi: \Gamma_{T} \rightarrow \mathbf{R}$, we consider the initial boundary value problem (IBVP)

$$
\begin{gathered}
L u=f(x, t, u, \nabla u), \quad(x, t) \in \bar{\Omega} \times(0, T], \\
u=\psi, \quad(x, t) \in \Gamma_{T},
\end{gathered}
$$


where $\psi$ is continuous and may be extended to $\bar{\pi}_{T}$ so as to belong to $C^{2+\alpha, 1+\alpha / 2}\left(\bar{\pi}_{T}\right)$, and satisfies compatibility conditions appropriate to (1), (2).

Given $\varphi: \mathbf{R}^{\boldsymbol{n}} \rightarrow \mathbf{R}$, we consider the Cauchy initial value problem (CIVP)

$$
\begin{gathered}
L u=f(x, t, u, \nabla u), \quad(x, t) \in \mathbf{R}^{n} \times(0, T], \\
u(x, 0)=\varphi(x),
\end{gathered}
$$

where again $\varphi$ satisfies appropriate compatibility conditions.

It will be clear from the development to follow that the results will also hold for IBVP's of the second kind.

A continuous function $v: \bar{\pi}_{T} \rightarrow \mathbf{R}$ is called a lower solution of (1), (2) in case

$$
v(x, t) \leqslant \psi(x, t), \quad(x, t) \in \Gamma_{T},
$$

and if for every $\left(x_{0}, t_{0}\right) \in \pi_{T}$ there exists an open neighborhood $U$ of $\left(x_{0}, t_{0}\right)$ and a finite set of functions $\left\{v_{r}\right\}_{1<r<s} \subseteq C^{2,1}\left(\bar{U} \cap \pi_{T}\right)$ such that

$$
L v_{r} \geqslant f\left(x, t, v_{r}, \nabla v_{r}\right), \quad(x, t) \in \bar{U} \cap \pi_{T}, 1<v<s,
$$

and

$$
v(x, t)=\max _{1<r<s} v_{r}(x, t), \quad(x, t) \in \bar{U} \cap \pi_{T} .
$$

If in the above definition the inequality signs in (5) and (6) are reversed and in (7) max is replaced by min then $v$ is called an upper solution of (1), (2).

One defines lower solutions for (3), (4) similarly, by replacing $\pi_{T}$ everywhere by $\mathbf{R}^{n} \times(0, T)$ and (5) by

$$
v(x, 0) \leqslant \varphi(x), \quad x \in \mathbf{R}^{n} .
$$

In analogous manner the concept of an upper solution for (3), (4) is defined.

If in any of the above definitions the inequalities occurring are replaced by strict inequalities the lower (resp. upper) solution will be called a strict lower (resp. strict upper) solution.

3. Invariance and existence. The first result needed in the development to follow is a known invariance result, a more general version of which appears in [3]. In the form given it is a Nagumo-Westphal type lemma.

LEMMA 1. Let $\alpha, \beta$ be strict lower and upper solutions of (1), (2) with $\alpha(x, t)<\beta(x, t),(x, t) \in \bar{\pi}_{T}$. If $u$ is a solution of (1), (2), then $\alpha(x, t)<$ $u(x, t)<\beta(x, t),(x, t) \in \bar{\pi}_{T}$.

Proof. Let $u \in C^{2,1}\left(\bar{\pi}_{T}\right)$ be a solution of (1), (2) and assume

$$
u\left(\bar{\pi}_{T}\right) \not \subset\left\{v: \alpha(x, t)<v<\beta(x, t),(x, t) \in \bar{\pi}_{T}\right\} \stackrel{\text { def }}{=}(\alpha, \beta) .
$$

We then may assume (other cases may be argued analagously) that theic exists $\left(x_{0}, t_{0}\right) \in \pi_{T}$ such that $u\left(x_{0}, t_{0}\right)=\alpha\left(x_{0}, t_{0}\right)$ and $\alpha(x, t)<u(x, t),(x, t)$ $\in \Omega \times\left[0, t_{0}\right)$. There exists a neighborhood $U$ of $\left(x_{0}, t_{0}\right)$ and functions $\left\{\alpha_{r}\right\}_{1<r<s}$ satisfying (6) and (7) with strict inequalities holding in (6). Let $\alpha_{k}\left(x_{0}, t_{0}\right)=\alpha\left(x_{0}, t_{0}\right)$, and put $v(x, t)=u(x, t)-\alpha_{k}(x, t)$, then $v(x, t)>0$, 
$(x, t) \in\left(\Omega \times\left[0, t_{0}\right)\right) \cap U, v_{t}\left(x_{0}, t_{0}\right)<0, \nabla v\left(x_{0}, t_{0}\right)=0$ and

$$
\sum_{i, j=1}^{n} a_{i j}\left(x_{0}, t_{0}\right) \frac{\partial v\left(x_{0}, t_{0}\right)}{\partial x_{i} \partial x_{j}} \geqslant 0
$$

and thus $(L v)\left(x_{0}, t_{0}\right) \geqslant 0$. But

$$
\begin{aligned}
(L v)\left(x_{0}, t_{0}\right) & =(L u)\left(x_{0}, t_{0}\right)-\left(L \alpha_{k}\right)\left(x_{0}, t_{0}\right) \\
& =f\left(x_{0}, t_{0}, u, \nabla u\right)-\left(L \alpha_{k}\right)\left(x_{0}, t_{0}\right) \\
& =f\left(x_{0}, t_{0}, \alpha_{k}\left(x_{0}, t_{0}\right), \nabla \alpha_{k}\left(x_{0}, t_{0}\right)\right)-\left(L \alpha_{k}\right)\left(x_{0}, t_{0}\right) \\
& <0, \text { by hypothesis. }
\end{aligned}
$$

We have thus arrived at a contradiction.

Using this invariance result we derive via an existence result from [3] the following existence theorem. A similar result is contained in Puel [13, Theorem 3.1, p. 97], though with a more complicated proof.

THEOREM 2. Let $\alpha, \beta$ be lower and upper solutions of (1), (2) respectively with $\alpha(x, t)<\beta(x, t),(x, t) \in \bar{\pi}_{T}$. Let there exist a positive continuous nondecreasing function $\Phi:[0, \infty) \rightarrow(0, \infty)$ such that $s^{2} / \Phi(s) \rightarrow \infty$ as $s \rightarrow \infty$ and

$$
|f(x, t, u, p)| \leqslant \Phi(|p|),
$$

$\alpha(x, t)<u<\beta(x, t),(x, t) \in \bar{\pi}_{T}$.

Then the IBVP (1), (2) has a solution $u \in C^{2,1}\left(\bar{\pi}_{T}\right)$ with $\alpha(x, t)<u(x, t)<$ $\beta(x, t),(x, t) \in \bar{\pi}_{T}$.

Proof. Define $\tilde{f}$ in the following way

$$
\tilde{f}(x, t, u, p)=\left\{\begin{array}{l}
f(x, t, \beta(x, t), p)+(u-\beta(x, t)), \quad \text { if } u>\beta(x, t), \\
f(x, t, u, p), \quad \text { if } \alpha(x, t) \leqslant u \leqslant \beta(x, t), \\
f(x, t, \alpha(x, t), p)+(u-\alpha(x, t)), \quad \text { if } u<\alpha(x, t) .
\end{array}\right.
$$

Choose constants $a$ and $b, a<0<b$, such that $a<\alpha(x, t)<\beta(x, t)<b$, $(x, t) \in \bar{\pi}_{T}$ and

$$
\tilde{f}(x, t, a, 0)<0<\tilde{f}(x, t, b, 0) .
$$

Using Theorem 2 of [3] we may conclude that the equation

$$
L u=\tilde{f}(x, t, u, \nabla u), \quad(x, t) \in \bar{\Omega} \times(0, T],
$$

has a solution $u$ satisfying (2) and $a<u(x, t)<b$. Let $\varepsilon>0$ be given. It follows from the definition of $\tilde{f}$ that $\alpha(x, t)-\varepsilon$ and $\beta(x, t)+\varepsilon$ are strict lower and upper solutions of (1), (2), respectively. Thus by Lemma 1 we have that $\alpha(x, t)-\varepsilon<u(x, t)<\beta(x, t)+\varepsilon, \quad(x, t) \in \bar{\pi}_{T}$. Since $\varepsilon>0$ was arbitrary, we conclude that in fact $\alpha(x, t) \leqslant u(x, t)<\beta(x, t),(x, t) \in \bar{\pi}_{T}$, and thus $u$ is a solution of (1), (2).

From the above existence theorem for IBVP's for (1), (2) we can easily derive the following existence theorem for the CIVP (3), (4). 
THEOREM 3. Let $\alpha$ and $\beta$ be lower and upper solutions, respectively, of (3), (4). For each $N>0$ let there exist a function $\Phi_{N}$ having the properties of $\Phi$ in Theorem 2 on the set $\bar{\pi}_{T}^{N}$. Then the CIVP (3), (4) has a solution $u \in C^{2,1}\left(\mathbf{R}^{n} \times\right.$ $[0, T])$ such that $\alpha(x, t) \leqslant u(x, t) \leqslant \beta(x, t),(x, t) \in \mathbf{R}^{n} \times[0, T]$.

Proof. For each positive integer $N$, there exists a solution $u_{N}$ of the IBVP

$$
\left\{\begin{array}{l}
L u=f(x, t, u, \nabla u), \quad(x, t) \in B^{N} \times(0, T], \\
u_{N}(x, 0)=\varphi(x), \quad x \in B^{N}, \\
u_{N}(x, t)=\theta_{N}(x, t), \quad(x, t) \in \partial B^{N} \times[0, T],
\end{array}\right.
$$

where $\theta_{N}$ is a sufficiently smooth function which satisfies

$$
\theta_{N}(x, 0)=\varphi(x), \quad \alpha(x, t) \leqslant \theta_{N}(x, t) \leqslant \beta(x, t),
$$

$(x, t) \in \bar{\Gamma}_{T}^{N}$, as follows easily by an application of Theorem 2. Using standard estimates and a diagonalization process one obtains a subsequence of $\left\{u_{N}\right\}_{N=1}^{\infty}$ which converges uniformly on compact subsets of $\mathbf{R}^{N} \times[0, T]$ to a solution of the CIVP (3), (4).

4. Maximal and minimal solutions. In this section we establish the existence of maximal and minimal solutions of the IBVP (1), (2) and the CIVP (3), (4). Mlak [7] has obtained such results for special classes of nonlinearities for $\operatorname{IBVP}(1),(2)$.

A solution $\bar{u}$ of the IBVP (1), (2) [CIVP (3), (4)] is a maximal solution relative to a given pair of lower and upper solutions $\alpha$ and $\beta$ with $\alpha(x, t)<$ $\beta(x, t),(x, t) \in \bar{\pi}_{T}\left[(x, t) \in \mathbf{R}^{n} \times[0, T]\right]$, if $\alpha(x, t)<\bar{u}(x, t)<\beta(x, t)$, and if $u$ is any other such solution then $u(x, t) \leqslant \bar{u}(x, t)$ for $(x, t) \in \bar{\pi}_{T}[(x, t) \in$ $\left.\mathbf{R}^{n} \times[0, T]\right]$. Minimal solutions are defined analagously.

THEOREM 4. Let the hypotheses of Theorem 2 hold. Then the IBVP (1), (2) has a maximal solution $u_{\max }$ and a minimal solution $u_{\min }$ relative to the pair $\alpha$, $\beta$.

Proof. Let $\mathcal{L}=\left\{v: \bar{\pi}_{T} \rightarrow \mathbf{R}, \alpha(x, t) \leqslant v(x, t) \leqslant \beta(x, t),(x, t) \in \bar{\pi}_{T}, v\right.$ is a lower solution of (1), (2)\}, and let $\mathscr{U}=\left\{w: \bar{\pi}_{T} \rightarrow \mathbf{R}, \alpha(x, t) \leqslant w(x, t) \leqslant\right.$ $\beta(x, t),(x, t) \in \bar{\pi}_{T}, w$ is an upper solution of (1), (2)\}. Define $u_{\max }$ and $u_{\min }$ by

$$
u_{\max }(x, t)=\sup \{v(x, t): v \in \mathcal{L}\}, \quad u_{\min }(x, t)=\inf \{w(x, t): w \in \mathcal{Q}\} .
$$

We shall establish that $u_{\max }$, thus defined, is a maximal solution, that $u_{\min }$ is a minimum solution will follow in much the same way.

Let $\left\{\left(x_{N}, t_{N}\right)\right\}_{N=1}^{\infty}$ be a countable dense subset of $\bar{\pi}_{T}$ and for $N=1$, $2, \ldots$, let $\left\{v_{N, m}\right\}_{m=1}^{\infty}$ be a sequence of lower solutions such that

$$
\lim _{m \rightarrow \infty} v_{N, m}\left(x_{N}, t_{N}\right)=u_{\max }\left(x_{N}, t_{N}\right) .
$$

Let $\alpha_{1}(x, t)=v_{1,1}(x, t)$; it follows from Theorem 2 that there exists a solution 
$u_{1}$ of (1), (2) such that $\alpha_{1}(x, t) \leqslant u_{1}(x, t) \leqslant \beta(x, t),(x, t) \in \bar{\pi}_{T}$. Let $\alpha_{2}$ be defined by

$$
\alpha_{2}(x, t)=\max \left\{u_{1}(x, t), v_{1,2}(x, t), v_{2,2}(x, t)\right\},
$$

then $\alpha_{2}$ is a lower solution of (1), (2) and by a further application of Theorem 2 there exists a solution $u_{2}$ of (1), (2) such that $\alpha_{2}(x, t) \leqslant u_{2}(x, t) \leqslant \beta(x, t)$, $(x, t) \in \bar{\pi}_{T}$. Inductively let $\alpha_{i+1}$ be the lower solution of (1), (2) defined by

$$
\alpha_{i+1}(x, t)=\max \left\{u_{i}(x, t), v_{1, i}(x, t), \ldots, v_{i, i}(x, t)\right\},
$$

where $u_{i}$ is a solution of (1), (2) such that $\alpha_{i}(x, t) \leqslant u_{i}(x, t) \leqslant \beta(x, t)$, $(x, t) \in \bar{\pi}_{T}$. We thus have, inductively, obtained a sequence of solutions $\left\{u_{i}\right\}_{i=1}^{\infty}$ of (1), (2) such that $\alpha(x, t) \leqslant u_{1}(x, t) \leqslant \cdots \leqslant u_{i}(x, t) \leqslant \cdots \leqslant$ $\beta(x, t),(x, t) \in \bar{\pi}_{T}$. Arguments similar to those used in the proof of Theorem 2 of [3] show that the sequence $\left\{u_{i}\right\}_{i=1}^{\infty}$ will converge to a solution $u$ of (1), (2). Furthermore it is clear that

$$
\lim _{i \rightarrow \infty} u_{i}\left(x_{N}, t_{N}\right)=u_{\max }\left(x_{N}, t_{N}\right)
$$

for $N=1,2, \ldots$ Hence $u\left(x_{N}, t_{N}\right) \geqslant v\left(x_{N}, t_{N}\right)$, for all $v \in \mathcal{L}$ and $N=1$, $2, \ldots$ and thus since $\left\{\left(x_{N}, t_{N}\right)\right\}_{N=1}^{\infty}$ is dense in $\bar{\pi}_{T}$ it follows that $u(x, t) \geqslant$ $v(x, t)$ for all $(x, t) \in \bar{\pi}_{T}, v \in \mathcal{E}$. Thus $u(x, t)=u_{\max }(x, t),(x, t) \in \bar{\pi}_{T}$ and consequently $u_{\max }$ is a maximal solution of (1), (2).

Using a similar argument and Theorem 3 one obtains the following result.

THEOREM 5. Let the hypotheses of Theorem 3 hold. Then the CIVP (3), (4) has a maximal solution $u_{\max }$ and a minimal solution $u_{\min }$ relative to the pair $\alpha$, $\beta$.

REMARK. It follows from Theorem 7 of [3] that the set of solutions $u$ of (1), (2) with $u_{\min }(x, t) \leqslant u(x, t) \leqslant u_{\max }(x, t),(x, t) \in \bar{\pi}_{T}$, is a continuum in $C^{1,0}\left(\bar{\pi}_{T}\right)$.

REMARK. The proof of Theorem 4 is based on a method due to Akǒ [1].

EXAMPLES. 1. The following example is similar to one given by Prodi [12] and Redheffer and Walter [14] to illustrate nonuniqueness for IBVP (1), (2). Consider

$$
\begin{gathered}
u_{x x}-u_{t}=-\left(u+(\cos x \cdot u)^{1 / 2}\right), \quad(x, t) \in(-\pi / 2, \pi / 2) \times(0,1), \\
u(x, 0)=0, \quad x \in(-\pi / 2, \pi / 2), \\
u(-\pi / 2, t)=0=u(\pi / 2, t), \quad t \in(0,1) .
\end{gathered}
$$

Then $\alpha(x, t) \equiv 0$ and $\beta(x, t)=t^{2}$ are lower and upper solutions, respectively, on $[-\pi / 2, \pi / 2] \times[0,1]$. The family $\left\{u\left(x, t ; t_{0}\right)\right\}$ given by

$$
u\left(x, t ; t_{0}\right)=\left\{\begin{array}{l}
0, \quad 0 \leqslant t \leqslant t_{0}, \\
\frac{\left(t-t_{0}\right)^{2} \cos x}{4}, \quad t_{0} \leqslant t \leqslant 1,
\end{array}\right.
$$


are solutions. By Theorem $4, u_{\max }$ and $u_{\min }$ exist between $\alpha$ and $\beta$ on $[-\pi / 2, \pi / 2] \times[0,1]$.

2. For the CIVP: $\Delta u-u_{t}=-u^{1-\gamma}, \gamma \in(0,1)$ with $u(x, 0)=0, \beta(x, t)$ $=(\gamma t)^{1 / \gamma}$ and $\alpha(x, t)=0$ are upper and lower solutions on $\mathbf{R}^{n} \times[0, T]$. By Theorem 5, $u_{\max }$ and $u_{\min }$ exist.

3. The next example is similar to one given by Mlak [8] for the IBVP. Consider $u_{x x}-u_{t}=f(x, u),(x, t) \in \mathbf{R} \times(0, \pi / 2), u(x, 0)=\cos x, x \in \mathbf{R}$, where

$$
f(x, u)=\left\{\begin{array}{l}
-u+\operatorname{sgn}(\cos x) \sqrt{\cos ^{2} x-u^{2}}, \quad|u| \leqslant \cos x, \\
-u, \quad|u| \geqslant \cos x .
\end{array}\right.
$$

Then $\beta(x, t)=3 e^{t}-1$ and $\alpha(x, t)=-3 e^{t}+1$ are upper and lower solutions, respectively. The family $\left\{u\left(x, t ; t_{0}\right)\right\}$ given by

$$
u\left(x, t ; t_{0}\right)=\left\{\begin{array}{l}
\cos x, \quad 0 \leqslant t \leqslant t_{0}, \\
\cos \left(t-t_{0}\right) \cos x, \quad t_{0} \leqslant t \leqslant \pi / 2,
\end{array}\right.
$$

are solutions of the given CIVP. By Theorem 5, $u_{\max }$ and $u_{\min }$ exist.

Remark. Prodi [12, p. 37] has noted that if $f$ satisfies a Hölder condition and a Nagumo growth condition with respect to $p$, then locally upper and lower solutions can be constructed. With this observation any problem (IBVP or CIVP) which has nonuniqueness locally has distinct maximal and minimal solutions respectively by our Theorems 4 and 5 .

\section{REFERENCES}

1. K. Akó, On the Dirichlet problem for quasi-linear elliptic differential equations of second order, J. Math. Soc. Japan 13 (1961), 45-62.

2. H. Amann, On the existence of positive solutions of nonlinear elliptic boundary value problems, Indiana Univ. Math. J. 21 (1971), 125-146.

3. J. W. Bebernes and K. Schmitt, Invariant sets and the Hukuhara-Kneser property for systems of parabolic partial differential equations, Rocky Mountain J. Math. 7 (1977), 557-568.

4. H. Fujita and S. Watanabe, On the uniqueness and nonuniqueness of solutions of initial value problems for some quasi-linear parabolic equations, Comm. Rare Appl. Math. 21 (1968), 163-652.

5. H. B. Keller, Elliptic boundary value problems suggested by nonlinear diffusion processes, Arch. Rational Mech. Anal. 5 (1969), 363-381.

6. O. A. Ladyzhenskaya, V. A. Solonnikov and N. N. Uraltseva, Linear and quasilinear equations of parabolic type, Transl. Math. Monographs, vol. 23, Amer. Math. Soc., Providence, R.I., 1968.

7. W. Mlak, Parabolic differential inequalities and the Chaplighin's method, Ann. Polon. Math. 8 (1960), 139-152.

8. An example of the equation $u_{t}=u_{x x}+f(x, t, u)$ with distinct maximum and minimum solutions of a mixed problem, Ann. Polon. Math. 13 (1963), 101-103.

9. M. Nagumo, On principally linear elliptic differential equations of second order, Osaka Math. J. 6 (1954), 207-229.

10. C. V. Pao, Successive approximations of some nonlinear initial-boundary value problems, SIAM J. Math. Anal. 5 (1974), 91-102.

11. Positive solutions of a nonlinear boundary value problem of parabolic type, J. Differential Equations 22 (1976), 145-163.

12. G. Prodi, Teoremi di esistenza per equazioni alle derivate parziali non lineari di tipo parabolico, Rend. Ist. Lombardo 86 (1953), 3-47. 
13. J. P. Puel, Existence comportement à l'infini et stabilité dans certaines problèmes quasilinéares elliptiques et paraboliques d'ordre 2, Ann. Scuola Norm. Sup. Pisa Cl. Sci. (4) 3 (1976), 89-119.

14. R. Redheffer and W. Walter, Counterexamples for parabolic differential equations, Math. $Z$. 153 (1977), 229-236.

15. D. H. Sattinger, Monotone methods in nonlinear elliptic and parabolic boundary value problems, Indiana Univ. J. Math. 211 (1972), 979-1000.

16. K. Schmitt, Boundary value problems for quasilinear second order elliptic equations, Nonlinear Anal. 2 (1978), 263-309.

17. F. Tomi, Über semilineare elliptische Differentialgleichungen zweiter Ordnung, Math. Z. 111 (1969), 350-366.

Department of Mathematics, University of Colorado, Boulder, Colorado 80309

Department of Mathematics, Univeristy of Utah, Salt Lake CtTy, Utah 84112 